\title{
Fractal Geometry of Urban Patterns and their Morphogenesis
}

\author{
PIERRE FRANKHAUSER \\ CNRS UPRESA 6049 THEMA, Université de Franche-Comté, F-25030 Besançon, France
}

(Received 25 December 1997)

\section{URBAN PATTERN FORMATION - SOME PRELIMINARY REMARKS}

One of the most striking features of urban dynamics is the emergence of highly irregular settlement patterns which are often perceived as amorphous. Urban planning deplores this evolution which turns out to be hardly controlable. Indeed the settlement dynamics is the result of complex socio-economic interaction processes including several levels of organization. On a microlevel, agents take decisions for constructing dwellings, shopping centers, manufactories, etc. Thus the settlement dynamics appears as a discrete process in time which should rather be interpreted as a random-like sequence of decisions. However these decisions are not taken in an independent way. On the one hand, there exist static physical constraints like mountain barriers, etc., on the other hand, planning policy defines the legal framework of settlement dynamics but guarantees also the construction and maintenance of infrastructure. In order to have an impact on planning decisions, agents organize themselves eventually in pressure groups with diverging aspirations. Moreover due to the expansion of cities, an increasing number of municipalities and other administrations are involved in these decisions and there exists a competition between different types of decision makers.

Nevertheless, despite of the complexity of the processes which contribute to the morphogenesis of these patterns and despite of their irregular form, they show some typical features like dendritic branches which grow along the transportation axes. Obviously this type of evolution seems to be a widespread phenomenon, which is even observed in countries with a centralistic planning policy. This inspires to interpret urban pattern formation, from a synergetic point of view, as a self-organization process where complex dynamics generates a certain type of spatial organization.

In order to analyze and to modelize this pattern formation, we should dispose of measures which allow to make evident their spatial organization. Indeed, a good knowledge about the urban morphology seems to be crucial for developing an "urban metric" in order to verify to what extend patterns simulated by some dynamic models are in concordance with real world patterns.

Traditional measures, used in urban planning, based on the notion of density, seem not to be 
suitable to characterize the morphology of such complex structures. Indeed, the density measures describe mean distributions (of population, builtup area, etc.) and refer thus, implicitly, to a homogeneous repartition of elements in space. Urban patterns are far away from such uniform distributions as it was already shown by several authors like Clark [11] or Bussière and Stovall [10]. We will show that a different approach, based on fractal geometry, turns out to be more suitable to describe such complex patterns.

\section{FRACTAL GEOMETRY - A NEW PARADIGM FOR URBAN MORPHOLOGY}

\subsection{Some Main Features of Fractal Geometry}

The analysis of complex morphology is a rather recent domain of research. Traditional theories and models based on linear approaches allowed not to generate complex forms as they are known e.g. in physics in turbulence, in diffusion processes or in dielectric breakdown phenomena, but also in biology or in texture analysis. It turned out that fractal geometry is a useful approach for describing complex forms. Moreover in some cases the results obtained by means of this approach allowed also to improve theoretical explanatory models.

The interest of fractal geometry results from different particularities. As pointed out Nicholis, fractals represent a new model of complex structures which are generated by rather simple mechanisms [24]. This remark refers to the inherent hierarchical feature of fractals which becomes obvious by the fact that the same type of geometric elements exists on a large range of scales. Nevertheless the organization of these patterns follows a well-defined, rather simple repartition law, the hyperbolic distribution. In our context it should be emphasized that such a law is well known in economics and urban geography as Paretodistribution. An example where such a relation is often observed is the rank-size distribution of cities which relates the population numbers of cities to their rank. Moreover, central place theory tackles with such a type of hierarchy, too, and other spatial systems, relevant for settlement dynamics, like transportation networks are also organized in a hierarchical way.

Under a geographic and urbanistic point of view it seems crucial, that fractals tackle with geometry. Thus the hierarchical law appears directly in the generated spatial patterns which consist of elements refering to a large range of scales. This renders a possibility to generate cluster hierarchies or complex boundaries with bulbes and bays of different size. This reminds us urban patterns: metropolitan areas are often constituted of a large number of clusters of quite different size and their boundaries show irregularities on a multitude of scales. Thus complexity of urban patterns seems closely related to the presence of spatial phenomena on different scales.

Recurring to measuring methods based on fractal geometry should allow to verify, to what extent real world patterns show such a type of hierarchy but also at which scale spatial organization changes or ruptures appear. Contrarily to usual filtering methods like convolutions, we may obtain a direct spatial interpretation of empirical results: if fractal pattern analysis makes evident that there exist ruptures in the spatial organization of the patterns, these ruptures may be localized on the pattern. This allows segmentation of zones with respect to their spatial organization. Moreover, we should be able to compare irregular empirical structures with regular reference patterns constructed by means of fractal geometry. Such comparison could allow to illustrate some features of the internal organization of the patterns.

By measuring hierarchy and characterizing thus a structure across the scales, fractal parameters transcribe another type of information than traditional density measures do. For example in a cluster distribution, a strong hierarchy would refer to a high concentration of mass in some big clusters and the presence of a rapidly increasing number of units with little mass. Such a local concentration of 
mass is contradictory to a homogeneous distribution where all elements should have the same size. Thus fractal parameters characterize the degree of concentration and of non-homogeneity.

We will now give concrete expression to the use of fractal geometry in matter of the modelization of urban pattern morphology by recurring to some particular approach, the Sierpinski carpets.

\subsection{The Sierpinski Carpet - a New Paradigm for Urban Patterns}

We will start the reflections about morphology of urban patterns by restricting ourselves to the formalization of their most elementary feature, the spatial repartition of the built-up area in space. This topic can be modelized in a convenient way by using Sierpinski carpets [14]. Figures 1-3 show some examples of such fractal objects. In Fig. 1 we see how a regular fractal is generated by a discrete iterative mapping procedure: a square of length $L$ represents the basic element. In a second step this element is reduced by a factor $r=1 / 3$ and $N=5$ of these small squares are arranged like a chess-board. Thus we have obtained $N_{1}=N$ elements of length $l_{1}=r L$. This procedure is repeated for each of the generated squares and thus the next figure is obtained. By going on with iteration we obtain

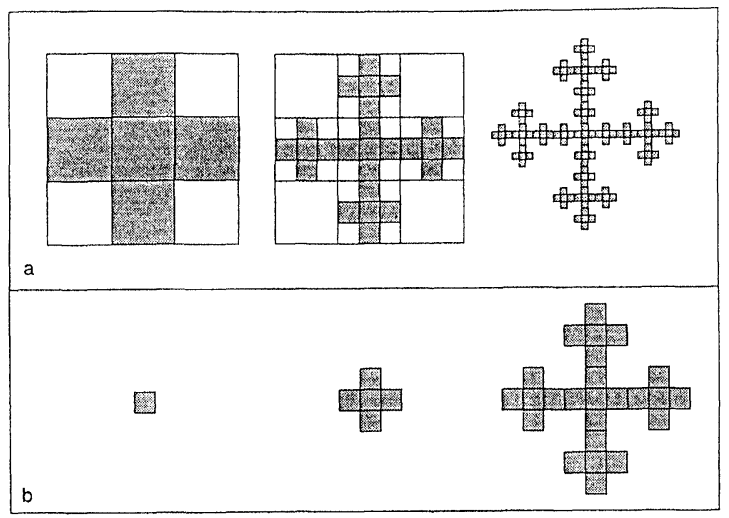

FIGURE 1 The generation of a Sierpinski carpet by iterative mapping using (a) a scaling factor $r=1 / 3$, the size of the squares tends to zero; (b) a scaling factor $r=1 / 3$, the size of the cluster tends to infinity. In (a) the lacunas, which appear in course of iteration, are indicated for the first two steps.

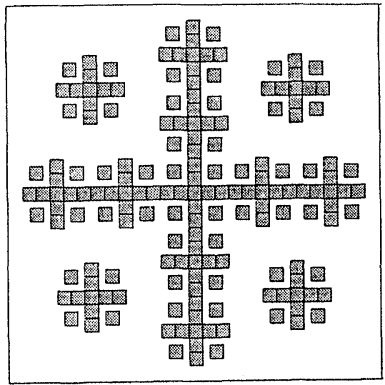

FIGURE 2 A Sierpinski carpet with hierarchical distribution of clusters $(r=1 / 5, N=13)$.

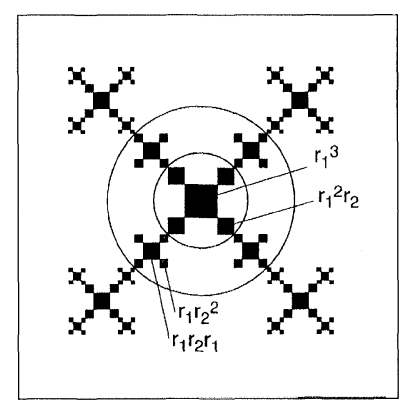

FIGURE 3 A multifractal Sierpinski carpet. Using two reducing factors $r_{1}=1 / 2$ and $r_{2}=1 / 4$ products of higher order of these factors occur in course of iteration. Thus a more complex hierarchy is obtained.

for some step $n$ :

$$
N_{n}=N^{n}, \quad l_{n}=r^{n} L
$$

Thus we observe that two complementary hierarchical systems occur:

- according to (1), at each step the number of elements increase, whereas their length diminishes. Thus we observe two long branches which intersect in the center, four smaller ones which intersect the long branches, etc.

- we observe, too, the emergence of another spatial hierarchy: in the immediate vicinity of the occupied (black) sites there exist many bays, whose size, for a given iteration step $n$ would be $\varepsilon=r^{n} L$. But there exist also larger bays of size $3 \varepsilon$ and finally even greater ones of size $9 \varepsilon$, etc., generated by the iteration steps before. Thus whole a hierarchy of lacunas exist (cf. Fig.1(a)). 
A consequence of this hierarchical organization is that the mass of the fractal, concentrated in the branches is distributed on space in a rather nonhomogeneous way. This implies that density measures loose their sense since they would hardly depend on the size of the reference area used and of the localization chosen. In particular by changing the size of the reference area, the obtained values would be crucially affected.

Other particularities occur when we try to apply other traditional measures like surface or length to fractal objects. Following the logic of the iteration we obtain for the surface at step $n$ :

$$
\begin{aligned}
A_{n} & =N_{n} \cdot l_{n}^{2}=N^{n} \cdot r^{2 \cdot n} \cdot L^{2} \\
& =\text { const } \cdot\left(\frac{5}{9}\right)^{n}, \\
& \Rightarrow \lim _{n \rightarrow \infty} A_{n}=0 .
\end{aligned}
$$

If surface vanishes we could estimate that the object is perhaps closer to linear topology. Thus we could estimate that we consider just the total extent, i.e. length $L_{n}$ of the fractal. It is easy to verify, that we would obtain

$$
\begin{aligned}
L_{n} & =\text { const } \cdot N^{n} \cdot r^{n}=\text { const } \cdot\left(\frac{5}{3}\right)^{n}, \\
& \Rightarrow \lim _{n \rightarrow \infty} L_{n}=\infty .
\end{aligned}
$$

Thus while the surface vanishes, the length of the object tends to infinity. The object seems to be longer than a one-dimensional object, but covers not the surface as well.

Let us just remind the reasoning which was introduced by Minkowski and Bouligand for measuring such type of sets. We require that there exists a measure $\mathcal{L}$ which should be constant all over the iteration. Obviously this holds neither for the perimeter nor for the surface. This requirement could be expressed by the following relation:

$$
N(\varepsilon) \cdot \varepsilon^{D} \stackrel{!}{=} \mathcal{L} .
$$

Here $N(\varepsilon)$ is the minimal number of elements, e.g. squares of size $\varepsilon$ which is necessary to cover the set. The exponent $D$, the fractal dimension, relates both the numbers and should guarantee that the relation holds even if we vary progressively the size of $\varepsilon$. For a constructed fractal we need just $N(\varepsilon)=N^{n}$ squares of base length $\varepsilon=r^{n} \cdot L$ to satisfy the condition that yields

$$
\left(N \cdot r^{D}\right)^{n}=\mathcal{L} \cdot L^{-D}
$$

If we scale our measure according to $\mathcal{L}=L^{-D}$, we obtain

$$
D=-\frac{\log N}{\log r}
$$

thus $D$ is really an invariant parameter with respect to iteration and its value is determined by the iteration parameters. In the present case we obtain $D=\log 5 / \log 3 \approx 1.47$.

If we apply the same logic to determine the dimension of a wholly occupied surface we would obtain the usual value $D=2$. In the same way it can be shown that an isolated point corresponds to $D=0$. For a line we would obtain $D=1$, but this value could also be obtained for a fractal set. In general we may conclude that low values of $D$ refer to a strong hierarchy whereas values close to $D=2$ are more close to homogeneity. But we should be aware that fractal dimensions do not take into account the morphological phenomena which refer to one unique scale. Thus $D=2$ would also be obtained for a chess-board since the lacunas have all the same size. However we may take into account topological properties by identifying the measure $\mathcal{L}$ to a generalized form factor $a$. Then we obtain, instead of relation (4):

$$
N(\varepsilon)=a \cdot \varepsilon^{-D} .
$$

We will now come back to the fractal of Fig. 1. Instead of reducing the initial square by a factor $r<1$ we will construct this pattern in another way (cf. Fig. 1(b)). We enlarge subsequently the figure by adding four copies of the initial squares at each of the sides. Then again four copies of the previously obtained figure are added at the tips of the cross. By repetition of this procedure the same arrangement is obtained as in the iteration 
previously discussed. We should however be aware that this generation "bottom up" instead of "top down" is mathematically only valid if we start from infinitesimal small squares.

This leads us immediately to a remark concerning the use of the concept for real world patterns. Even if we assume an organization of patterns which follows a fractal law, we could not expect that we reach scales where the size of the elements vanishes. In reality it makes no sense for urban patterns to tackle with details which refer to a scale lying beyond the mean size of buildings since their interior plan is of minor importance in the context considered. For such real world situation Mandelbrot introduced the notion of "prefractals". Since fractal behavior may change across the scales we prefer to speak of scaling behavior, like physicists do.

The structure of Fig. 1 is constituted of a solely ramified cluster with an interior hierarchy. Figure 2 shows that it is also possible to generate a cluster hierarchy when some of the elements are placed separately from the central cluster. Finally Fig. 3 shows a conceptual enlargement. In this case two different scaling factors $r_{1}$ and $r_{2}$ has been used and thus, in higher iteration steps, elements are generated by factors of the type $r_{1}^{k} \cdot r_{2}^{n-k}(k=$ $0,1, \ldots, n)$ combining different orders of $r_{1}$ and $r_{2}$. Using such a generating law a higher degree of complexity is reached and we obtain a multifractal pattern $[17,20]$. In those sets, the fractal behavior is no longer uniform but depends on the site chosen. It is possible to determine a series of fractal dimensions which refer to higher order correlations of occupied sites. Then the number of pairs, triples, etc. of an occupied point lying closer together than a given distance $\varepsilon$ is considered. For uniform fractals all these dimensions have the same value, for multifractals the range within these dimensions vary, and may be considered as a measure for the complexity of the pattern.

By means of a multifractal formalization, it is also possible to modelize a nonuniform, hierarchical distribution of mass on a pattern. E.g. we assume to assign to each of the generated squares at the first iteration step a part of a mass $p_{i}(i=$ $1,2, \ldots, N), \Sigma_{i} p_{i}=1$. In the next iteration steps mass factors are obtained, constituted of multinomial combinations of the factors $p_{i}$. Such an approach may serve to describe the population repartition in a city.

Let us now use the concept of Sierpinski carpets in order to make evident how a link can be established to urban patterns. We assume that the iteration has been realized up to a step $n$ and we interpret a square as a simplified cartographic representation of a building. The Sierpinski carpet should then be a rough model of a settlement. We could assume that there exist two main road axes which intersect in the center of the cluster. A lot of buildings are concentrated along these two perpendicular axes. This seems quite reasonable since in many cases settlements grow along existing road axes what allows minimization of the costs of constructing and undertaking infrastructure. However there exist also the lateral branches mentioned above. Buildings situated on these axes are farther away from the main road axis, but they are closer to the initial center of the settlement than in the case they would have been localized on the tips of a purely cross-like cluster. Another advantage of this arrangement results from the fact that all buildings are close to a border of the cluster and therefore to the lacunas which we may interpret as green areas. The arrangement seems to be a good compromise between the desire to be at the same time close to the city center and to recreation areas. Indeed there exist some plans of urbanists who recur to such a logic in order to assure that dwellings may benefit simultaneously of the vicinity of different types of services. Figure 4 shows such an example, a plan proposed by Hilberseimer for the reconstruction of Chicago [22] which shows regular fractal behavior. The goal of this plan was the interconnection of all dwellings with a ramified green area and a transportation network. Obviously a hierarchical concept, where both the types of land use penetrate each other, turned out to be a suitable solution for this aim. Fractal geometry could thus become a reference to reflect about the spatial arrangements 


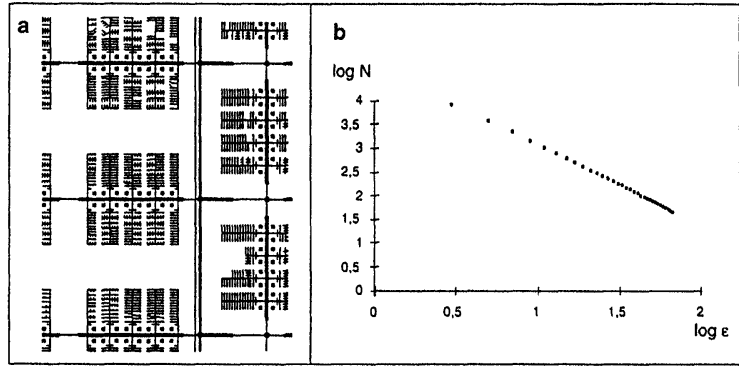

FIGURE 4 The plan of Hilberseimer for the reconstruction of Chicago. On the right hand the dilatation analysis of the structure. The slope of rather regular straight line corresponds to a dimension of $D \approx 1.68$.

which optimize simultaneously different requirements of accessibility [19]. Moreover if real patterns show such complex morphology, this may perhaps be a hint that agents tend to optimize their residential choice according to similar criteria $[14,19]$.

However if we are interested in the analysis of real world patterns we should be aware that such highly symmetric forms are not realistic. Indeed cities are certainly not issued from an iterative mapping procedure, but according to our preliminary reflections they are rather the result of a sequence of individual decisions which generate highly irregular patterns. Thus it seems more suitable to recur to a random-like description of urban dynamics. ${ }^{1}$ Indeed it is possible to introduce random elements in the mapping procedure by changing the position and eventually the number added at each step in such a way that statistically the fractal behavior is not affected. The obtained results reminds more urban structures than regular fractals. The possibility to compare random fractals with regular structures as the plan of Hilberseimer allows to reflect to what extend real urban structures correspond to such multicriterial optimization strategies.

If the introduction of random elements in the mapping procedure allows to simulate more realistic patterns we may ask if the iteration may be interpreted as a dynamic process of pattern generation. We may of course interpret the iteration as a sequence of time steps $\Delta t$. However, according to the generating law, we implicitly assume then a particular type of dynamics, since the number of elements $N_{n}=N^{n}$, increases according to a geometric progression:

$$
\frac{\Delta N_{n}}{\Delta t}=N^{n-1} .
$$

We remind that in a time-continuous interpretation this corresponds to a exponential growth. Even if such a type of dynamics seems like sudden urban growth not completely irrealistic we should not restrict to such law. But this is indeed not necessary; it is possible to simulate the emergence of fractal patterns formation as a subsequent random-like addition of elements.

\section{HOW TO MEASURE FRACTALITY}

Since real world pattern are not directly related to a mapping procedure, the fractal behavior must be tested by some measuring methods. For this aim different algorithms have been developed which refer to the logic of covering enounced previously: by means of some reference length $\varepsilon$ which is subsequently varied, the number of elements $N(\varepsilon)$ is counted necessary to cover all occupied sites. For fractal structures there exists a relation analogous to (7):

$$
N(\varepsilon)=a(\varepsilon) \cdot \varepsilon^{-D} .
$$

The form factor $a(\varepsilon)$ may depend on the chosen algorithm but can also transcribe local deviations from fractal law. For interpretation of empirical data it is convenient to recur to a double-logarithmic representation of this relation

$$
\log N(\varepsilon)=\log a(\varepsilon)-D \cdot \log \varepsilon .
$$

Indeed we get aware that we obtain now a linear relation where the slope value is just the fractal

\footnotetext{
${ }^{1}$ We remind that some authors conceived models based on a probabilistic approach, but not referring to fractal geometry (cf. e.g. [25]). The fractal urban simulation model of Batty [3], which will be discussed later on, tackles with random processes, too.
} 
dimension. Moreover deviations from a linear relation may be detected easily.

There exist several algorithms based on relations like (10). Some of these methods give a general information about the repartition of mass, e.g. the built-up area, within a given zone. We speak then of global methods of analysis. In urban analysis three of these methods were particularly used: the grid analysis, the dilation analysis and the correlation analysis [5,14,16,28]. A rather different approach is the radial analysis which transcribes the spatial organization in the vicinity of a chosen counting center and which is thus a local analysis.

If urban patterns were rather simple fractal structures, it could be expected that the same values are obtained by using different methods. However it cannot be excluded, that urban patterns are more complex, i.e. that there exist local variations of fractal dimensions, i.e. a multifractal behavior. Then the information obtained by different methods turn out to be complementary. Thus the scaling exponent obtained by the radial analysis may be interpreted as the LipschitzHölder-exponent which is related in a more complex way to the series of fractal dimensions mentioned above $[7,12,18]$. The series of higher order correlation dimensions can be obtained directly by counting the number of pairs, triples, etc. in the vicinity of an occupied site or by means of the grid analysis (cf. next section). In any way the scaling behavior is again studied by subsequently varying $\varepsilon$.

In order to analyze urban patterns, usually cartographic representations are digitized and the counting procedures are done by a computer program.

\subsection{Global Methods of Analysis}

The most simple way to study fractal behavior is the grid analysis. A zone to be analyzed is selected and covered by a grid. Then the number of grid elements which contain at least one occupied site is determined. The grid distance is subsequently varied, and at each step, again the number of occupied grid elements is counted. Thus, according to (9); the relation between the number of occupied elements $N(\varepsilon)$ and the corresponding grid distance $\varepsilon$ is obtained. In many cases the double-logarithmic representation of this relation shows a very regular shape, in particular when using cartographic representations at a little scale which are only reliable for studying the spatial organization on the scale of metropolitan areas, since details of intraurban structure are neglected.

This method is also used in multifractal analysis. Instead of distinguishing just empty and occupied grid elements, the real mass is taken into account for each grid element, what allows to determine higher order correlation dimensions.

The grid analysis shows sometimes ambiguities which can be avoided by using the dilation analysis. This algorithm corresponds to the method Minkowski proposed for studying such sets under the viewpoint of measure theory. In this case each occupied site is surrounded by squares whose base length is subsequently enlarged. The whole surface of these squares is considered as occupied. Thus the squares referring to sites which lie close together intersect first, and in course of iteration more and more huge clusters appear. In order to obtain the number of squares of length $\varepsilon$ which is necessary to cover the total occupied surface at iteration step $n$, we divide at each step this total occupied surface by the surface $\varepsilon^{2}$ of the squares. Then an analogous relation to (9) is obtained. This method has e.g. been used for testing fractal behavior of the plan of Hilberseimer (Fig. 4). For urban patterns usually rather regular empirical curves are obtained with both the global methods. ${ }^{2}$

\subsection{The Radial Analysis}

It turned out that this method plays a particular role in urban pattern analysis. From a mathematical point of view this type of analysis is rather

\footnotetext{
${ }^{2}$ For rather coarse grained grid values all grid elements still contain occupied points and we obtain $D=2$. However beyond a welldefined threshold fractal behavior occurs.
} 
different from the previous ones. The information obtained refers directly to a chosen site and describes the mass distribution around this center. For this aim the center is surrounded by a circle of radius $\varepsilon$ and the number of occupied sites $N(\varepsilon)$ is counted within this circle. ${ }^{3}$ The radius $\varepsilon$ is gradually enlarged and for each step the number $N(\varepsilon)$ is determined. A relation equivalent to (9) allows us to estimate the scaling exponent which we designate by $\alpha$, since it corresponds rather to the Lipschitz-Hölder-exponent, as pointed out before.

Due to its local character the curves obtained by this type of analysis are less regular than the one obtained by global methods. In order to make better evident the informations transcribed by this type of analysis, it turned out that another type of representation of the results is more suitable $[5,16]$. Instead of the fractal relation itself the sequence of local slope values $\alpha_{i}^{(\mathrm{loc})}$ of the double logarithmic representation is depicted as a function of the distance $\varepsilon_{i}$ from the counting center:

$$
\alpha_{i}^{(\mathrm{loc})}=\frac{\log N\left(\varepsilon_{i}\right)-\log N\left(\varepsilon_{i-1}\right)}{\log \varepsilon_{i}-\log \varepsilon_{i-1}} .
$$

For a perfect fractal relation the values $\alpha_{i}^{(\text {loc })}$ should be constant all over the range of distances. But even for regular, constructed fractals such fluctuations exist since the logic of the counting algorithm is not in concordance with that of the iterative mapping procedure. For urban patterns, we may usually distinguish two types of deviations:

- local deviations from the fractal law. They traduce the presence of huge lacunas, i.e. free places like parks or, respectively, big clusters as important buildings. Such deviations may be attributed to a local variation of the form factor $a=a(\varepsilon)$ since mean scaling behavior is not affected;

- real changes of the mean slope value. Beyond a critical distance another well-defined value is observed over a sufficiently large range of distances. This shows a rupture of the scaling

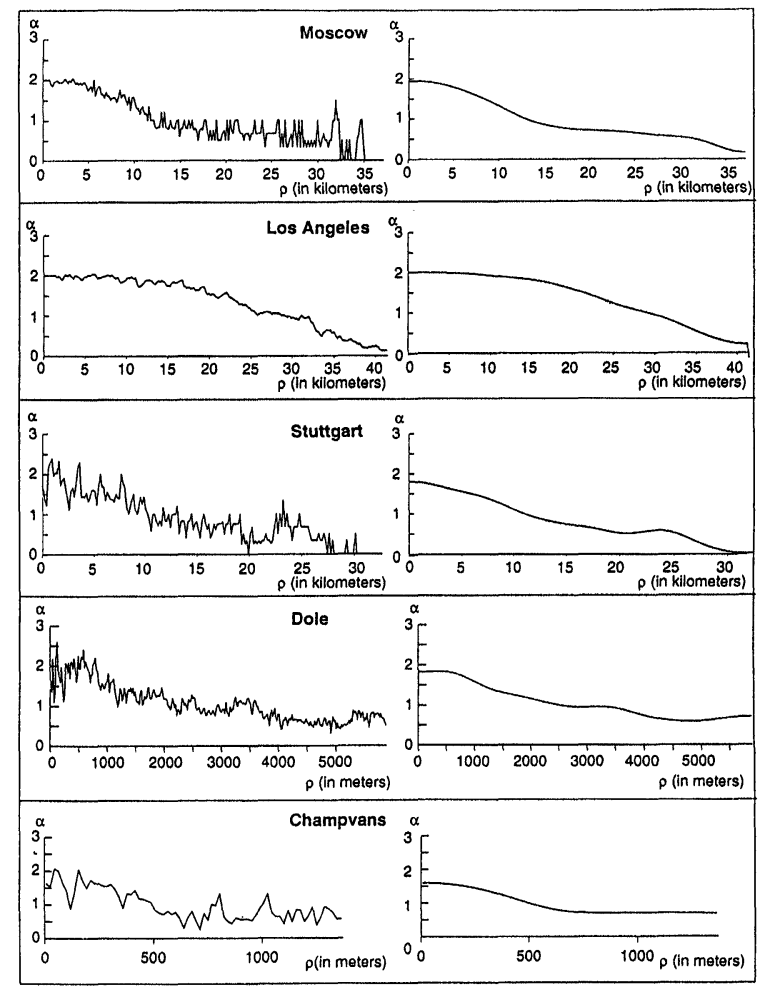

FIGURE 5 A comparison of the scaling behavior of agglomerations of different size: on the left the original curves, on the right the smoothed curves.

behavior, i.e. the pattern shows another kind of spatial organization beyond a critical distance what yields $\alpha=\alpha(\varepsilon)$.

Figure 5 shows such curves. We observe both the the phenomena enounced. For the ruptures the corresponding distance may easily be detected and thus be localized on the urban pattern (cf. Fig. 6). Moreover the signal fluctuates around a dominant mean behavior what refers to the local perturbations.

It may be surprising that there exist values $\alpha_{i}^{(\text {loc) }}>2$, i.e. values which exceed the value obtained for a homogeneously occupied surface. However it can be shown [18] that if the slope depends on the distance, the parameter $\alpha_{i}^{(\mathrm{loc})}=\alpha_{i}^{(\mathrm{loc})}\left(\varepsilon_{i}\right)$ must be interpreted in another way. Indeed by using a continuous interpretation

\footnotetext{
${ }^{3}$ For computer analysis instead of a circle a square is used, what affects is just the prefactor in the fractal relation.
} 


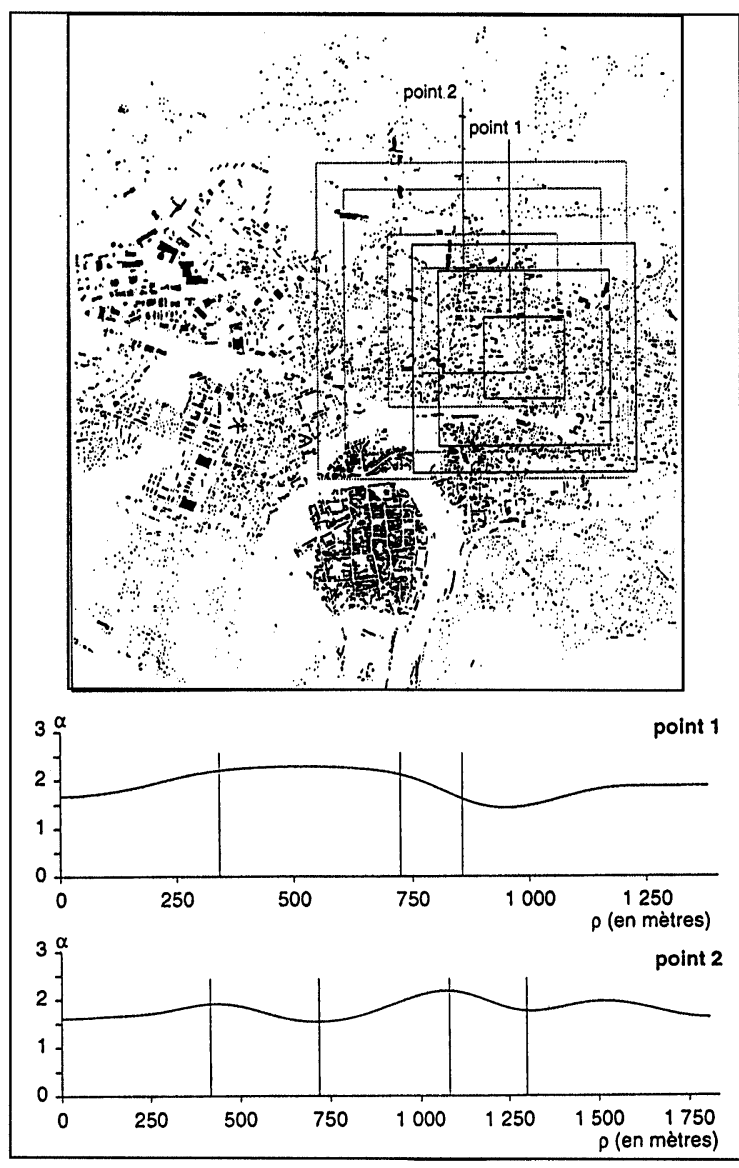

FIGURE 6 The smoothed curves referring to two counting centers in the North of the peripheric zone of Besançon. On the cartographic representation used for the analysis, the frames corresponding to the ruptures identified on the curves are indicated. We observe that some of the sides of these frames are close one to the other.

of $\alpha_{i}^{(\text {loc })}$

$$
\alpha^{(\mathrm{loc})}=\frac{\mathrm{d} \log N}{\mathrm{~d} \log \varepsilon} \equiv \lim _{\log \varepsilon_{i} \rightarrow \log \varepsilon_{i-1}} \alpha_{i}^{(\mathrm{loc})},
$$

we obtain

$$
\frac{\mathrm{d} \log N}{\mathrm{~d} \log \varepsilon}=\frac{\mathrm{d} \log a}{\mathrm{~d} \log \varepsilon}+\log \varepsilon \frac{\mathrm{d} \alpha}{\mathrm{d} \log \varepsilon}+\alpha \equiv \alpha^{(\mathrm{loc})} .
$$

Other than the local value of $\alpha$, two other terms appear. According to previous remarks the first term may be associated to the local perturbations, whereas the second one describes the long range structural changes. Using a terminology close economics we may design $\alpha_{i}^{(\text {loc })}$ as marginal scaling exponent.

In order to make evident the real ruptures in the patterns, we used Gaussian smoothing to eliminate local fluctuations (cf. Figs. 5 and 6).

\section{FRACTAL INVESTIGATIONS OF SETTLEMENT PATTERNS}

In matter of analyzing urban patterns, fractal geometry was first used for studying the relation between the amount of built-up surface and the length of their boundary. If we assume, in concordance with the geometry of Sierpinski carpets, that the occupied surface as well as the boundaries follow a fractal law we obtain the relation

$$
A(\varepsilon)=\mathrm{const} \cdot P^{D^{(\text {surf })} / D^{\text {(pri) }}}
$$

In their first investigations Batty and Longley [5] referred to a simplified relation, where they assumed that the intra-urban space may be considered as completely occupied, so that $D^{\text {(surf) }}=2$. Then fractality refers only to the tortuous aspect of the boundary whose scaling behavior is measured by use of the "coastline of Britain analysis". In this case the length of the boundary is measured by a yardstick of varying length $\varepsilon$ and fractal dimension is obtained by a relation analogous to (9). Such a point of view is justified if we use simplified cartographic representations at a scale of $1: 100,000$ or even smaller ones which neglect details on intra-urban scale. Then usually patterns are obtained which look like ink-dots. For the boundaries Batty and Longley obtained values which lie in the range $D=1.2-1.5$.

Recurring to a similar type of cartographic representation but according to the relation (14) we determined for a set of cities the occupied area $A_{i}$ and the perimeter $P_{i}$. We observed that the plot of the pairs $\left(\log P_{i}, \log A_{i}\right)$ lies on a straight line where the slope is close to one. This yields 
$D^{\text {(surf) }} \approx D^{\text {(peri) }}$ in concordance with Sierpinski carpet geometry.

Another preliminary analysis concerned the rank-size distribution of the settlement clusters within metropolitan areas. For this aim we introduced surface size classes and counted the number of clusters which belong to these classes. For European cities and some North-American cities we obtained a rather regular Pareto-distribution which confirms the fractal character of the repartition of built-up area on this global scale and thus a strong hierarchical organization. On the contrary, American agglomerations for which growth has been dominated early by the motorization showed no developed hierarchy.

Some authors dealt also with the link between fractal geometry and central place theory, first by Arlinghaus and Arlinghaus [2]. The particular question of the repartition of the built-up area in the scheme of Christaller has been considered by Frankhauser [14] and, recently, François gave a fractal interpretation of population distribution under the aspect of central place theory [17].

\subsection{The Data Base Considered}

Above all, fractal investigations of settlement patterns focussed on metropolitan areas and referred to a regional level $[5,14]$. For this aim the previously mentioned type of simplified cartographic representations has been used. Until now more than 20 metropoles have been analyzed. The set of cities we considered consisted of European, North-American, Australian and some Third World cities.

During the last years different investigations were realized recurring to data bases which contain informations up to the microscales of buildings. Thus it became possible to study the spatial organization of cities on an intra-urban level. Whereas Batty and Xie [6] used an American data source, the "tiger files", we analyzed a certain number of agglomerations in the East of France on the base of topographic maps of $1: 50,000$ and $1: 25,000$ as well as GIS data ${ }^{4}[15,16]$. In view of the high amount of data, we restricted until now to smaller cities which lie in the range of 26,000 120,000 inhabitants. Moreover, a certain number of villages were analyzed lying in the hinterland of these cities and one particular investigation tackled with whole the settlement system in a rather rural region. ${ }^{5}$

In the following we should give a brief survey on the obtained results, in order to make evident what kind of information fractal analysis provides.

\subsection{The City Centers - the Natural Centers of Symmetry?}

For comparing the scaling behavior of agglomerations it turned out that interesting informations are obtained by using the radial analysis and by choosing the center of the agglomeration as counting center. First of all we observe that a similar shape is obtained for the curves more or less independently of the scale we consider. Figure 5 shows a comparison of the curves of scaling behavior for agglomerations at different scales. On the left the original signal is represented, whereas the right column shows the smoothed curves. The main features of the curves may be summarized as follows:

- Close to the counting center we observe usually that the values of the scaling exponent remain on a rather constant level $\alpha=1.8-1.9$. This zone refers to the historical center of the cities.

- Follows a transient zone where the scaling exponent drops, what should be interpreted as hint that the spatial organization changes. For European agglomerations this decrease occurs within a rather restricted range of distances, whereas for the more homogeneous patterns of American cities the decrease of the scaling

\footnotetext{
${ }^{4}$ The maps at $1: 25,000$ are, by their cartographic conception rather close to reality, whereas the information is less reliable for smaller scales.

${ }^{5}$ These investigations were in part realized by J. Prost, L. Gauguel, S. Lhomme and G. Vuillamy in the frame of their masters thesis.
} 
exponent extends to a wide range. In Fig. 5 this may be verified for the curve obtained for Los Angeles (cf. Section 4.3). This behavior refers to the peripheric ring of the cities.

- When the border of the central cluster is reached, we observe for European cities usually a rather extended zone for which the scaling exponent remains rather constant; i.e. a well-defined fractal behavior occurs. The scaling exponent varies around $D \approx 1.6$. This zone corresponds to the suburban area.

- Finally another zone of decrease is observed when the border of the agglomeration is reached.

In general using coarse grained cartographic representations fluctuations around the dominant scaling behavior are less important than for data bases obtained by topographic maps, since neglecting details operates like a smoothing filter. Moreover the presence of huge buildings or parks may disturb the curve in the vicinity of the counting center. It seems more surprising that the same type of result is obtained for huge agglomerations like Moscow or Stuttgart but also for small towns of about 30,000 inhabitant and even for villages. This should be interpreted as hint that there exists a rather general law for the decrease of the ratio of built-up area in direction of the town border. Indeed we observe that in many cases the morphology of these settlements shows some similarities: beyond a rather compact center of the city, there exist some dendritic built-up branches along the main transportation axes. Such a morphology occurs for large cities as well as for villages.

However in some cases we obtain the same type of curve even for other types of patterns. This is the case for Besançon where topological conditions have hardly influenced the emergence of the urban pattern. Thus the city growed in a fan-shaped way. Nevertheless the obtained curve of scaling behavior is rather similar to cities like Lyons or Moscow what shows that the main decay of density is in concordance with the usual rules.

These results are not trivial, since we observe at different scales also cases which show particular forms of the curves. We mentioned already the case of Los Angeles. On the level of medium cities the conurbation of Montbéliard is a rather interesting case. This agglomeration must be considered as an aggregate of several towns of comparable size. Moreover the area is dominated by the most important motor factory of Peugeot. Thus very different results are obtained with respect to the city center choosen. For example for Audincourt, lying in the heart of the conurbation, only a slight decline of the curve of scaling behavior is observed when reaching the border of the city. However by increasing the counting radius one enters immediately a neighboring agglomeration and the scaling exponents augments again and reaches approximately the previously observed level. Such minor variations of the scaling exponent appear too for greater distances. Thus whole the agglomeration could more or less be considered as a unique cluster with a dominant mean scaling behavior and some local deviations.

If counting centers are chosen in the centers of towns lying more in the periphery of the conurbation and close to the enounced factory rather particular curves are obtained which are more similar to those of peripheric zones (cf. next section). On the contrary, if the Peugeot manufactory is taken as counting center, the curve of scaling behavior resembles more to those of city centers, despite of the fact that this manufactory lies in the periphery of the conurbation, too. Obviously this enterprise has become an important symmetry center within the pattern since a similar shape of curve is observed like that of city centers. This may be explained by the fact that this enterprise has played an important role in course of urbanization; indeed a large amount of dwellings were constructed in its neighborhood. In this context another example turns out to be interesting, a new city build up in suburbs of Besançon. The goal of the planners was to create a rather autonomous entity with respect to the historical center. Despite of the rather different architecture consisting of large blocks, the curve obtained corresponds again to those of city centers. Thus the intention of 
planning appears through the pattern organization. This makes also obvious that fractal analysis provides a powerful instrument to compare planned cities with irregular settlement patterns.

\subsection{Towards a Typology of Agglomerations}

The previous discussion of the curve obtained for city centers showed that fractal analysis could serve to identify a particular class of urban patterns on rather different scales. Thus we may expect that such type of analysis could serve to classify urban patterns, but the restricted number of patterns analyzed until now allows not yet to propose a general typology. Nevertheless we should give an example how information obtained could be used for such a purpose. Recurring to maps of $1: 500,000$ we could distinguish the following types of patterns, by using several counting algorithms and comparing the results [14]:

- For European and some North-American cities the grid dimension obtained lies in the range 1.60-1.73. The lower values are obtained for cities where there exists a great contrast between the central cluster and the hinterland, like at Moscow. Then a stronger hierarchy may be expected. The lowest value were observed for St. Petersburg (1.38) and Stuttgart (1.41). The pattern of the Stuttgart region is particularly fragmented and there exist agglomerations of rather different size. Indeed the rank-size analysis of the clusters made evident that there exists a rather regular hierarchical organization, what holds for St. Petersburg, too. The values obtained for the radial analysis referring to the historical centers lie between 1.94 and 1.99. Low values refer to a rather strong decrease of the built-up share with respect to the distance of the center. Thus Moscow and Berlin show such a behavior. Indeed both the agglomerations show a dendritic structure which may be explained by the important role which suburban railway played for the growth process. Some North-American agglomerations showed the same behavior.
- A completely different behavior was observed for some American and Australian cities for which the growth process was early influenced by motorization. This holds e.g. for Los Angeles and for Melbourne. For these agglomerations the grid dimensions as well as the scaling exponents obtained by radial analysis are close to two, what indicates that the pattern is rather homogeneous.

Another promising possibility is to recur to the shape of the curve of scaling behavior in order to distinguish different types of spatial organization. Preliminary investigations for a rural region in the East of France, the Bresse, has shown that different types of curves may be obtained according to the morphology of the patterns.

\subsection{Other Domains of Application}

The example of the city centers discussed in the previous section showed what kind of information about urban pattern may be obtained by using fractal analysis of the built-up area. We want to complete this presentation by giving a survey over other potential domains of application in matter of the investigation of urban structures.

\section{Delimitation of Urbanized Areas and Intra-Urban Ruptures}

The curves of scaling behavior can be used in order to localize the ruptures in the urban patterns. For this aim the distance from the counting center are identified where scaling behavior changes and square-like frames may be drawn on the cartographic representation referring to these distances (Fig. 6). It is then possible to identify on the patterns the perimeter if urbanization on the only base of urban morphology. This is of interest since the urban perimeter is often fixed by means of administrative definitions.

The example of the Montbéliard region has shown that there exists other types of curves as they are usually obtained for the symmetry centers of urbanization. Figure 6 shows the results 
obtained for three counting centers localized in the Northern part of the peripheric ring of Besançon, a rather complex zone with respect to its spatial organization. Thus for each counting point several ruptures appear which has been identified on the pattern. However we get aware that some of the sides of these frames are close, one to the other, and transcribe thus the same change in the spatial organization of the pattern. By varying subsequently the position of the counting center we may expect that it may be possible to trace border lines which separates town sections according to their spatial organization.

There exist also other methods, based on the grid analysis to make ruptures evident in urban pattern. In this case the center of the window is subsequently moved and the dimension is determined. By coding the value of the dimension by gray levels, e.g. black for $D=2$ and white for $D=0$, it is possible to assign the corresponding gray value to the area around each counting point. Thus a dimension map is obtained which allows to detect zones with the same scaling behavior and to localize ruptures [19].

\section{Fractal Representation of Urban Patterns}

As pointed out above, the fact that fractals tackle with geometry, could be rather interesting for the comparison of urban patterns with regular reference structures which show a similar hierarchical organization. However we should be aware that fractal dimensions characterize hierarchy in a rather global way: when defining the fractal dimension by means of the iterative mapping procedure solely the number of the reduced elements came into play and not their position. In order to respect striking features of the "metric" of urban patterns we should however take into account the distribution of different types of lacunas. Two types of lacunas seem to be of particular interest for urban patterns:

- the hierarchy of free space like parks, squares and courtyards;

- the hierarchy of the street system.
Preliminary investigations for several town sections of Paris have shown that there may exist rather regular hierarchies in street systems by representing the relation between total length of streets with respect to their breadth [14]. Taking into account the results of such types of analysis, we could expect that rather interesting fractal transcriptions of urban patterns could be possible. Figure 7 gives just an idea how such a reference pattern could be constructed. In this case the system of lacunas respects in a only qualitative way the presence of squares, courtyards and streets in the real world pattern.

\section{The Analysis of Population Distribution}

Fractal analysis has also been used to analyze the repartition of the population. Batty [4] and François [17] studied by means of radial analysis the decrease of population with respect to a given center. Whereas Batty referred to the scale of agglomerations, François considered the whole french system of cities. By choosing Paris as counting center, she observed a similarly regular shape of curve as we obtained for the built-up area when considering counting points localized in the city centers.

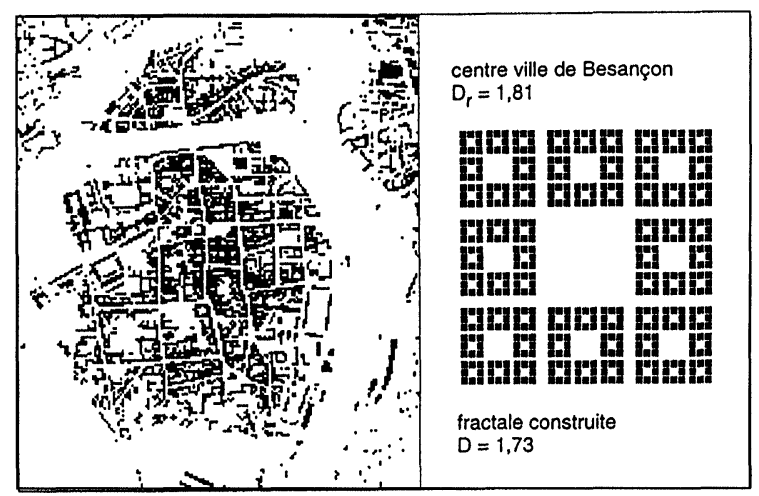

FIGURE 7 The city center of Besancon $(D \approx 1.81)$ and a qualitative fractal transcription of the pattern $(D=1.73)$. The distribution of the lacunas have been chosen in such a way that some characteristic shapes of the pattern appear, in particular the street system and the rather great courtyards in the center of the blocks of houses. 
Le Bras $[7,8]$ used a quite other approach, based on a multifractal model, for simulating the repartition of population on a rather regional scale. He recurred to an iterative mapping procedure, similar to that one we used for generating complex Sierpinski carpets: space is subdivided in a grid and to each grid element a certain ratio of the population $p_{i}$ is attributed, which correspond to the different scaling factors. In the following steps, the grid elements becomes finer, but due to the mapping algorithms, again mixed terms composed of multiples of the different factors $p_{i}$ appear and thus a complex distribution of population may be represented. According to the multifractal theory a link may be established to the corresponding Lipschitz-Hölder exponents. Recently Appelby [1] used a similar approach in order to analyze the population distribution in a systems of cities.

For analyzing the intra-urban scale also nonoccupied space should be taken into account according to the Sierpinski carpet approach. It is indeed possible to combine both the aspects by introducing simply a factor $p_{0}=0$ which creates the lacunas in course of iteration [18]. This formalization allows also to measure multifractal behavior when disposing of population data on a rather fine level of resolution. Then you may attribute a certain amount of population to each occupied pixel and the multifractal analysis provides the series of fractal dimensions. We recurred to this method for analyzing the population repartition in different town sections of Besançon. The results obtained showed that the range of dimensions is larger for quarters with a complex pattern consisting of individual housing and huge blocks than for sections where you find only one particular type of houses. However for new town sections consisting of blocks, the dimensions are lower than in old sections. Indeed for the first ones we observe a great contrast between a high concentration of population in the blocks surrounded by large green areas whereas old centers show a more homogeneous patterns where houses have only some flats and where the courtyards are small. ${ }^{6}$

We imagine that by considering the height of the buildings we may describe the roughness of the surface of a city by using a multifractal formalization in a similar way. Such analysis is expected to be useful in matter of modelizing the propagation of pollution in cities. Recently Woloscyn [30] proposed to use a multifractal approach for describing the roughness of buildings in the streets, in order to analyze noise propagation.

\section{FRACTAL APPROACHES OF URBAN PATTERNS FORMATION}

\subsection{Some Empirical Results}

We saw already that the choice of commuting modes highly influence the urban morphogenesis. We remind the examples of Berlin or Moscow where the role played by public transportation networks was crucial. On the contrary the pattern of Los Angeles is different since the early motorization leads to a more homogeneous spatial organization. In order to get more insight in the dynamics of pattern formation, it should be instructive to analyze urban patterns over time. Figure 8 shows time sequences of the curves of scaling behavior for Lons-le-Saunier and Berlin. Despite of the fact that these agglomerations are of rather different size, we observe a similar tendency in the evolution of the curves. For the first date the decrease of the scaling exponent is rather strong. Moreover there exist some local variations for greater distances which refer to important aggregates in the suburban area. For the following periods the decrease of the scaling exponent is less abrupt, since the transient zone between center and suburban area becomes more extended. In the same time the local variations of the scaling exponent in the suburban zone disappear, the curve becomes more smooth. We may interpret such an evolution as a hint that the central agglomeration dominates more and more the

\footnotetext{
${ }^{6}$ These preliminary investigations were in part realized by $\mathrm{T}$. Thevenin in the frame of his masters thesis.
} 


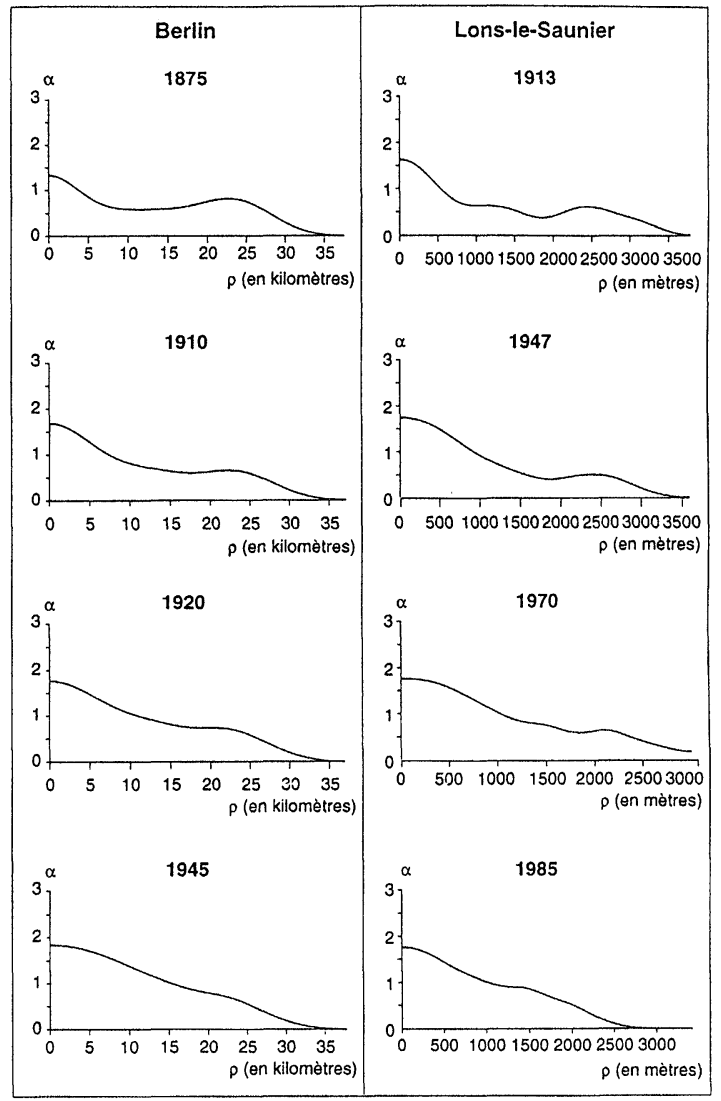

FIGURE 8 The comparison of the growth of Berlin and Lyons by means of the smoothed curves of scaling behavior. The same type of transformation of the shape of the curves occur.

spatial organization of the suburban zone and that the settlements loose their independence by merging in a larger cluster. Under a synergetic point of view we may then speak of an enslaving principle. ${ }^{7}$

This interpretation is confirmed by choosing the center of suburban villages as initial points for counting. The example of Montmorot (Fig. 9) shows that at a first time ruptures exist between the main agglomeration, Lons-le-Saunier and this village. At the second date these ruptures have

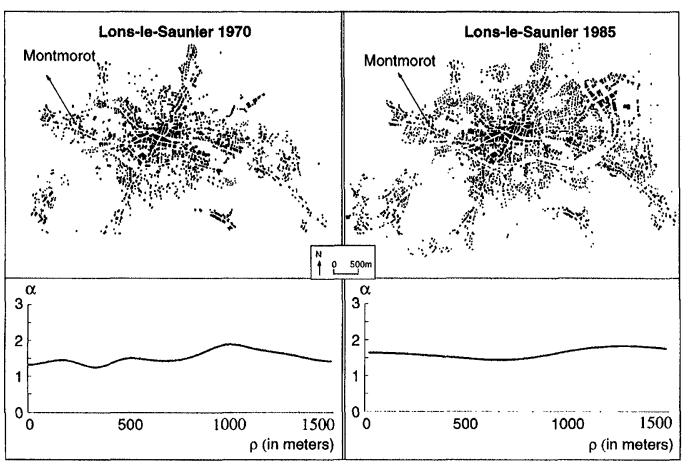

FIGURE 9 The scaling behavior of Montmorot near Lonsle-Saunier at two periods. Due to the urbanisation a common

disappeared, and a nearly constant scaling behavior is observed. Since radial analysis corresponds, by definition, at each given distance to an integration over whole the range of angles, the obtained result traduces not just the fact that space between both the agglomerations is subsequently filled, but also that urbanization progressed in whole the vicinity of Montmorot. Thus a unique cluster with a common scaling behavior seems to emerge.

In this context another result is of interest. By considering the curve of scaling behavior before smoothing it may be observed that the fluctuations around the dominant behavior diminishes in course of urbanization. Figure 10 shows the evolution of a agglomeration near Montbéliard, which was, at the first date, just a hamlet. Thus a very irregular signal is observed and no scaling behavior at all can be detected. In course of time a certain number of dwellings were build up in the vicinity and we get aware that the fluctuations diminish, whereas a well-defined mean scaling behavior occurs. We may summarize that obviously urban morphogenesis generates a certain type of spatial organization even if the patterns in question are often perceived as irregular.

Obviously the curve of scaling behavior, and in particular the ratio between mean signal and noise traduces the degree of interior organization of the

\footnotetext{
${ }^{7}$ Weidlich and Haag [26] proposed a synergetic model of sudden urban growth, which tackles with the emergence of a metropole growing on the detriment of other cities. More recently the P.A.R.I.S research team developed a multi-agent model which reveals, too, the development of a city system. However, both the approaches refer to a more regional scale and consider rather demographic and economic aspects than urban morphology [21].
} 


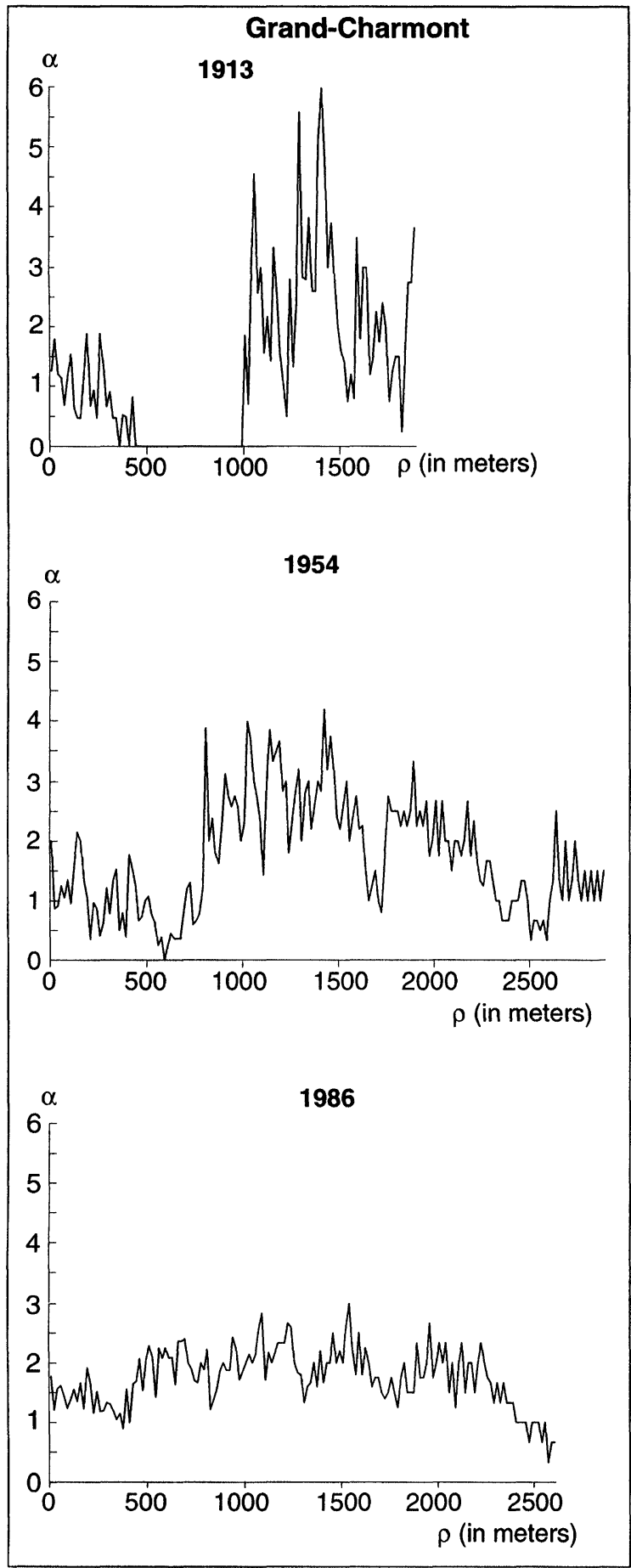

FIGURE 10 The non-smoothed curve of scaling behavior of Grand Charmont near Montbéliard for three periods. In course of urbanization the fluctuations are attenuated and a clear mean behavior appears. pattern. By interpreting urban pattern formation as self-organization process, we may conclude that this curve plays a similar role as do order parameters in phase transitions.

\subsection{Fractal Models of Urban Growth}

The results obtained by fractal analysis encouraged several authors to simulate urban growth by recurring to fractal modeling approaches. These models are based on enlarged concepts of cellular automata which allow to generate complex patterns by well-defined rules. Cellular automata are grid-like arrangements of cells each of which belongs to an attribute class, represented e.g. by colors. These cells may change their attribute according to some rules, which refer usually solely to the next neighbor cells. These rules are in principle deterministic but may include random processes. In the present context these cellular automata are used as a cartographic representation of space. Each cell corresponds to an area of a certain size according to the cartographic resolution assumed. The attributes refer to different types of land use. Urban dynamics is perceived as a discrete series of change in land use. In fact in some models several cells change their attribute simultaneously at one time step.

In particular Batty [3,5] and Makse [23] recurred to modeling approaches close to them used in physics to describe diffusion limited aggregation or dielectric breakdown. These are domains where random cellular automata are largely used to generate fractal structures. Batty used some morphological growth parameters to calibrate such a model in order to get good conformity between simulated patterns and observed ones. The patterns obtained seem rather satisfying with respect to their comparison to real world morphology. Nevertheless we should be aware that such approaches are rather descriptive, what holds, of course, in general for the use of fractals. Indeed no real explanatory link is established, e.g. between the location strategies of agents and the growth probabilities. 
Another type of approach tends to modelize directly spatial interaction by use of cellular automata. These models refer rather to contagion models as they are used in biology $[9,13]$. Under this point of view we should mention that Makse introduced in his model the influence of the neighborhood of a site for defining a probability to be urbanized, but another model, conceived by White and Engelen [27,29] goes beyond: the authors introduce for each site a potential for the transition from one type of land use to another one. These potentials describe observations about the choice behavior of agents. Thus, e.g. the vicinity of an industrial area is unfavorable to the implantation of a residential zone. Recently more economic topics has been integrated in the modeling concept. The deterministic transition rules are based on the enounced potential functions, which are however modified by a noise function. One difficulty is that, due to the highly complex dynamics, it is still difficult to evaluate the influence of this noise function on pattern formation. All the models use fractal measure to compare generated pattern to empiric ones.

Anyway, this type of modeling approach remains promising, since space is included in a more explicit way than in traditional concepts. Thus urban pattern formation can be directly controlled by means of mophological analysis. ${ }^{8}$

\section{CONCLUSION}

We have seen that using fractal geometry for the description of urban pattern morphology implies a different approach of space. The traditional point of view is based on the assumption of a homogeneous repartition of elements in space, as it is traduced by density measures, whereas fractal geometry refers to a hierarchical repartition law, corresponding to the hyperbolic distribution. Such a law is well known in economics and in urban geography, e.g. the central place theory tackles with such a hierarchy. On an intra-urban scale, street networks, squares, etc. are hierarchically organized, too. Moreover for the built-up area the radial decrease of density is also a well-known phenomenon. Thus the use of such an approach seems adapted to analyze urban morphology in different contexts.

Since fractal geometry refers to hierarchies, this approach suits to analyze to what extend a spatial hierarchies exist which are perhaps veiled by noise. Indeed fractal measures describe the degree of hierarchy and thus of concentration of spatial elements in clusters. Due to its hierarchical character, the fractal approach allows an investigation across the scales. The empirical investigations showed that, on the one hand, particular shape of curves occurs for many centers of quite different size, on the other hand it is possible to identify particular scales where the spatial organization changes. The geometric character of the approach allows to localize in a convenient way ruptures in the patterns.

Moreover different kinds of patterns may be distinguished at each scale, so that fractal analysis could contribute to pattern classification. These results make also evident the link between morphology and settlement dynamics. Deeper investigation of the pattern evolution showed that the urbanization process is closely related to the emergence of an internal structure which is usually characterized by a growing dominance of the main aggregate and a decrease of fluctuations in the empirical curves.

The analogy between the methods of measure and the generation of patterns provides also the possibility to compare real world patterns with constructed reference patterns based on fractal geometry or with patterns simulated by fractal growth models. ${ }^{9}$

\footnotetext{
${ }^{8}$ Sigg et al. [25] elaborated recently a rather interesting model which tends to simulate pattern formation by means of a stochastic dynamic approach.

${ }^{9}$ In this context we should emphasize that fractal geometry is until now the only geometric implication of a distribution law, there exists e.g. no geometry which refers to log-normal distribution, another well-known law in human geography.
} 
Future research activities should focus on the introduction of complementary measures based on the concept of spatial hierachy which allow a more detailed description of the spatial arrangements of the elements. The multifractal concept seems promising, too: the possible range of applications reaches from the analysis of social segregation up the determination of the roughness of the surface of the built-up area in order to study the propagation of pollution.

A quite other domain refers to urban planning. As pointed out fractal patterns could, due to their complex structures, better assure the simultaneous proximity to different types of services or land uses as simple compact structures. General conceptual reflections could help to obtain criteria in what concrete case fractal patterns really correspond to optimal solutions.

\section{Acknowledgements}

The author is particularly grateful to Denise Pumain, Lena Sanders, France Guérin and AnneMarie Odouze for many fruitful discussions as well as to Joelle Maillardet and Thomas Thevenin for the realization of the illustrations. The research has been supported by the CNRS and the PIR-ville project.

\section{References}

[1] S. Appelby. Multifractal characterization of the distribution pattern of the human population. Geographical Analysis, (2), 1996.

[2] S.L. Arlinghaus and W.-C. Arlinghaus. The fractal theory of central place geometry: a diophantine analysis of fractal generators for arbitrary löschian numbers. Geographical Analysis, 21(2), 1989.

[3] M. Batty. Cities and fractals: simulating growth and form. In A.J. Crilly, R.A. Earnshaw and H. Jones, editors, Fractals and Chaos, 1991.

[4] M. Batty and K.-S. Kim. Form follows function: reformulating urban population density function. Urban Studies, 29(7), 1992.

[5] M. Batty and P. Longley. Fractal Cities, a Geometry of Form and Function. Academic Press, 1994.

[6] M. Batty and Y. Xie. Preliminary evidence for a theory of fractal cities. Environment and Planning A, 28, 1996.
[7] H. Le Bras. La planète au village. DATAR, éditions de l'aube, 1993.

[8] H. Le Bras. Le peuplement de l'Europe. DATAR, 1996.

[9] A. Bunde et al. Universality classes for spreading phenomena: A new model with fixed static but continuously tunable kinetic exponents. Physical Review Letters, 55(7) 1985.

[10] R. Bussière and T. Stovall. Systèmes évolutifs urbains et régionaux à l'état d'équilibre.

[11] C. Clark. Urban population densities. Journal of the Royal Statistical Society, Series A, 64, 1951.

[12] J. Feder. Fractals. Plenum Press, 1988.

[13] P. Frankhauser. Fractal aspects of urban systems. In Sonderforschungsbereich 230 "Natürliche Konstruktionen", editor, Beiträge zum I. Internationalen Symposium des SFB 230 Natürliche Konstruktionen - Leichtbau in Architektur und Natur Teil 1, 1988.

[14] P. Frankhauser. La fractalité des structures urbaines. Anthropos, 1994.

[15] P. Frankhauser. Echelles, hiérarchies et fait urbain. Habilitation à diriger des recherches, Université de Franche-Comté, 1996.

[16] P. Frankhauser. L'analyse fractale, un nouvel outil pour l'analyse spatiale des tissus urbains. In J.-B. BocquetAppel, D. Courgeau and D. Pumain, editors, Spatial Analysis of Biodemographic Data. John Libbey and INED, 1996. Série Congresses and Colloquia.

[17] N. François. Méthode d'application de la géométrie fractale à la description des systèmes de villes et des réseaux de transport, 1997. Thèse de Doctorat, Université Paris 1.

[18] P. Frankhauser. L'analyse fractale, un nouvel outil de réflexion dans l'analyse spatiale des agglomérations urbaines. Population, 4, 1997.

[19] P. Frankhauser and C. Genre-Grandpierre. La géométrie fractale, un nouvel outil d'analyse et de réflexion pour l'investigation des réseaux de transport, 1997. XXIIIe Colloque de l'ASRDLF, Lille.

[20] J.-F. Gouyet. Physique et Structures Fractales. Masson, 1992.

[21] F. Guérin-Pace, H. Mathian, D. Pumain, L. Sanders and S. Bura. SIMPOP, un modèle multi-agents pour l'évolution du système de peuplement. In Acte des Premières Rencontres Theoquant, 1995.

[22] L. Hilberseimer. Entfaltung einer Planungsidee. Ullstein, 1963.

[23] A. Makse, S. Havlin and H.E. Stanley. Modeling urban growth pattern. Nature, 377, 1995.

[24] G. Nicholis. Symmetriebrüche und die Perzeption von Formen. In M. Baudson, editor, Zeit-die vierte Kategorie in der Kunst, 1985.

[25] T. Sigg, N. Koch and W. Weidlich. Urban evolution in interaction with population dynamics. In J.-B. BocquetAppel, D. Courgeau and D. Pumain, editors, Spatial Analysis of Biodemographic Data. John Libbey and INED, 1997. Série Congresses and Colloquia.

[26] W. Weidlich and G. Haag. A dynamic phase transition model for spatial agllomeration process. Journal of Regional Science, 1986.

[27] R. White. A cellular automata approach to the evolution of urban land use patterns. In Paper presented at the 7 th European Colloquium on Theoretical and Quantitative Geography, Stockholm, 1991. 
[28] R. White and G. Engelen. Cellular automata and fractal urban form: a cellular modelling approach to the evolution of urban land use patterns. Environment and Planning A, 25, 1993.

[29] R. White and G. Engelen. Urban systems dynamics and cellular automata: Fractal structures between order and chaos. Chaos, Solitons and Fractals, 4(4), 1994.
[30] P. Woloscyn. Vers un simulateur des ambiances sonores urbaines: le modulateur morpho-accoustique. Les Annales de la Recherche Urbaine, 4, 1996. 


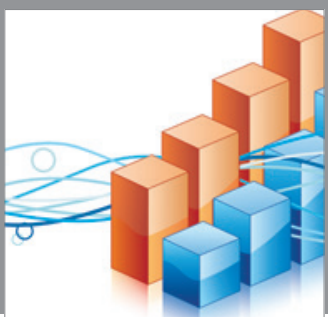

Advances in

Operations Research

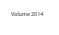

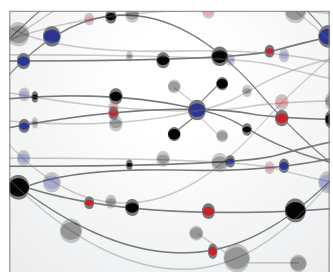

\section{The Scientific} World Journal
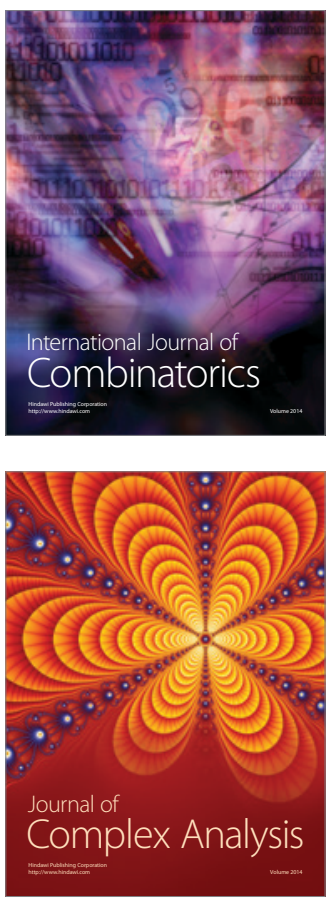

International Journal of

Mathematics and

Mathematical

Sciences
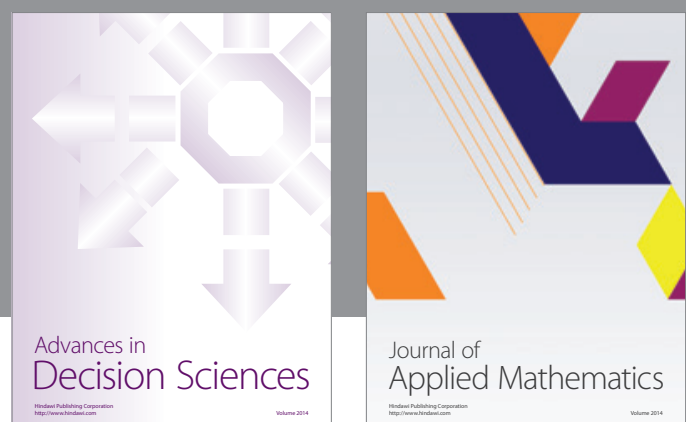

Journal of

Applied Mathematics
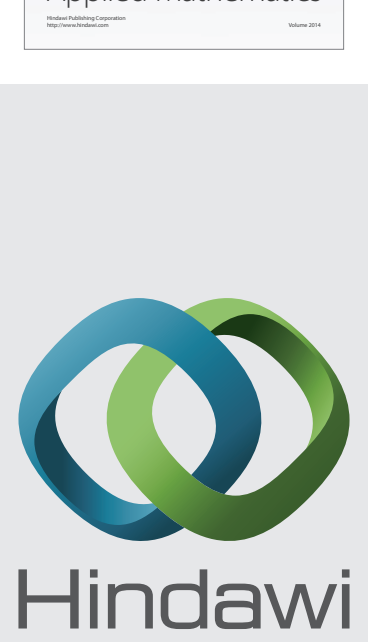

Submit your manuscripts at http://www.hindawi.com
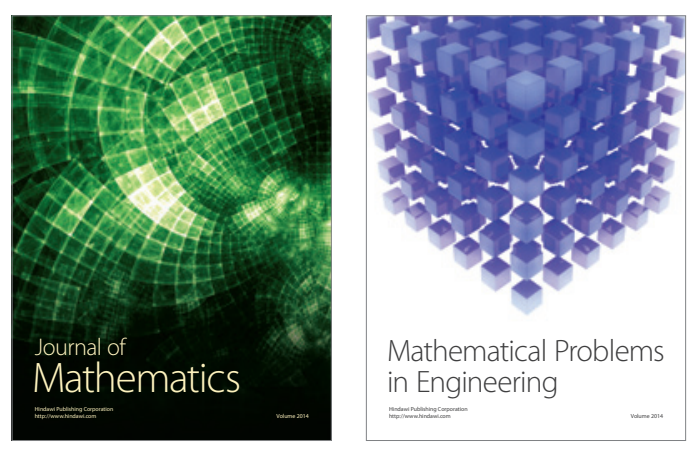

Mathematical Problems in Engineering
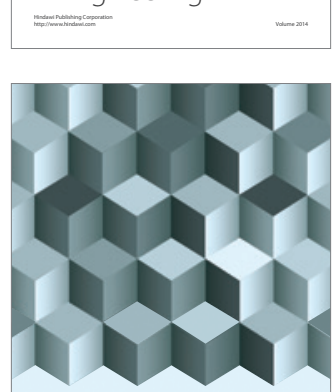

Journal of

Function Spaces
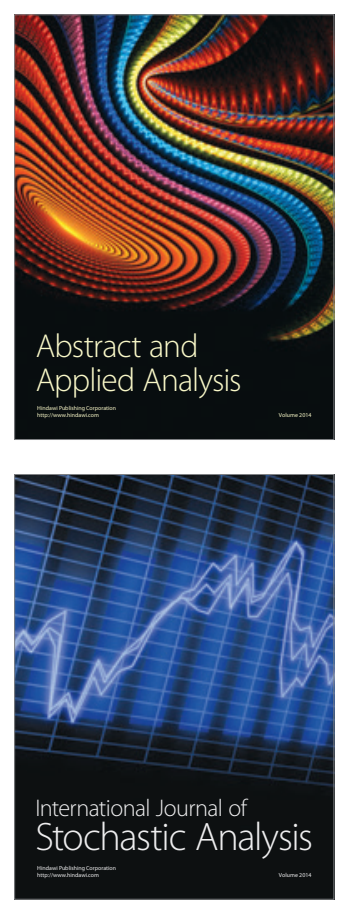

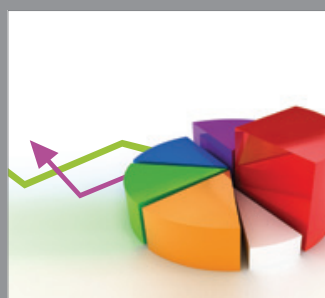

ournal of

Probability and Statistics

Promensencen
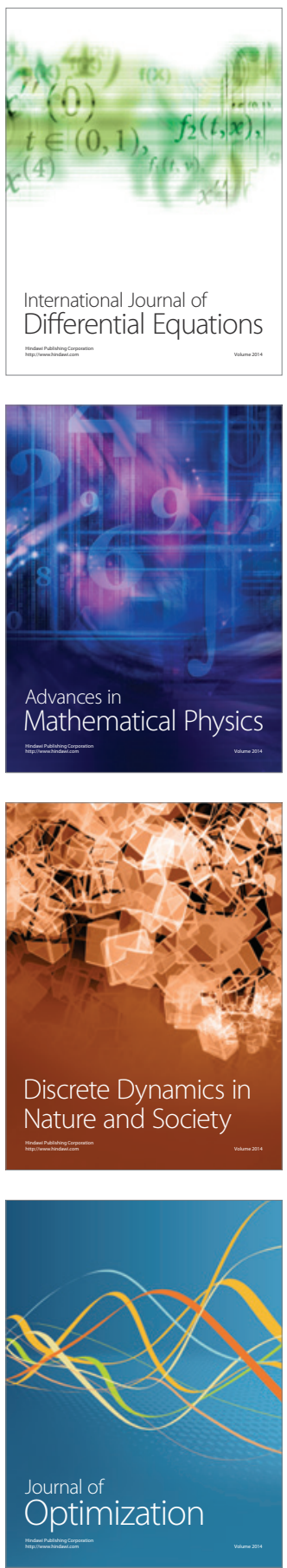